satisfactory as a candidate for clues to recognize FM in patients with non-rheumatic diseases, as a diagnosis of FM ultimately is made by a physician.

Disclosure of Interests: : None declared

DOI: 10.1136/annrheumdis-2019-eular.7576

\section{THU0478 IS FIBROMYALGIA ASSOCIATED WITH STRUCTURAL OR FUNCTIONAL ABNORMALITIES IN SKELETAL MUSCLE?}

Carlos Pineda ${ }^{1}$, Héctor García ${ }^{1}$, Gabriela Pineda ${ }^{1}$, Angélica Peña ${ }^{2}$, Laura Aline Martínez ${ }^{3}$, Jaime Mendoza Torres ${ }^{1}$, Luis Carlos Rodriguez Delgado ${ }^{1}$ Mariana Moreno ${ }^{4}$, Jessica Gutierrez ${ }^{1}$, Araceli Bernal ${ }^{1}$, Marwin Gutierrez ${ }^{1}$, Manuel Martinez-Lavin ${ }^{3} .{ }^{1}$ Instituto Nacional de Rehabilitación Luis Guillermo Ibarra Ibarra, Mexico City, Mexico; ${ }^{2}$ IMSS Hospital General Regional No. 1, Querétaro, Mexico; ${ }^{3}$ Instituto Nacional de Cardiología Ignacio Chávez, Mexico City, Mexico; ${ }^{4}$ ISSSTE Hospital General Zona Fernando Quiroz, Mexico City, Mexico

Background: Fibromyalgia (FM) is a chronic nonarticular pain syndrome of unknown etiology characterized by diffuse muscular pain, fatigue and mood disturbances. Previous studies have shown absence of skeletal muscle degeneration, regeneration or inflammation. Altered muscle fiber size distribution and decreased capillary density were the only abnormalities reported.

From the clinical point of view some FM symptoms (fatigue, pain, myalgias, trigger points, stiffness) suggest skeletal muscle involvement.

Objectives: The objective of this study is to determine if there are structural and functional abnormalities in the skeletal muscle in patients with primary FM assessed by means of a non-invasive, low-cost multimodality approach.

Methods: Female patients > 18 years, with FM diagnosis (ACR 2010 criteria) and healthy controls matched by age. Skeletal muscle morpho-structure and function were assessed by: body mass index (BMI), total fat mass and total muscle mass calculated by direct segmental multi-frequency bioelectrical impedance analysis. Cross-sectional measurements of rectus femoris muscle area and tissue echogenicity were evaluated by ultrasound and pixel analysis. Maximum handgrip strength by digital dynamometry, gait speed (6-meter time walk test), and FM Health Assessment Questionnaire (FHAQ).

Results: A total of $94 \mathrm{FM}$ patients and 140 healthy controls were included, mean age was 51.8 years +/- 10.8 vs 50.2 +/- 11.3, respectively $(p=0.27)$. FM patients compared with controls had similar BMI $\left(27.9 \mathrm{~kg} / \mathrm{m}^{2}+/-4.9\right.$ vs $\left.26.8+/-4.5, \mathrm{p}=0.14\right)$, higher total body fat mass $(27.8 \mathrm{~kg}+/-9.2$ vs $25.1+/-7.6, p=0.04)$; rectus femoris muscle area was also higher for FM patients $\left(44.6 \mathrm{~cm}^{2}+/-11.4\right.$ vs $41.7+/$ 13.9, $p=0.05$ ); regarding ultrasound tissue echogenicity, FM patients demonstrated higher mean brightness in rectus femoris (157.2 pixels +/19.4 vs $149.9+/-22.4, \mathrm{p}=0.01)$; lesser handgrip strength $(22.0 \mathrm{~kg}+/$ 6.6 vs $26.2+/-5.4 p=0.0001)$; slower gait speed $(1.14 \mathrm{~m} / \mathrm{s}+/-0.2$ vs $1.3+/-0.2 \mathrm{p}=0.0001$ ); and major impairment in daily activities by FHAQ $(47 \%$ vs $2.90 \% p=0.0001)$

Conclusion: This non-invasive multimodal evaluation of the skeletal muscle showed the presence of structural and functional abnormalities in women with FM. These changes can be attributed to sedentary and hypoactive lifestyle with consequent higher total body fat mass, skeletal muscle fat infiltration, and functional impairment of activities of daily living Alternatively, these abnormalities may also be an expression of a smallfiber polyneuropathy (myoneurovascular dysregulation). Further studies are required to elucidate its underlying pathological process.

\section{REFERENCES:}

[1] Lodahl M, Treister R, Oaklander AL. Specific symptoms may discriminate between fibromyalgia patients with vs without objective test evidence of small-fiber polyneuropathy. Pain Rep 2018; 3(1): e633.

[2] Martínez-Lavín, M. Fibromyalgia and small fiber neuropathy: the plot thickens. Clin Rheumatol 2018;37: 3167.

Disclosure of Interests: None declared

DOI: 10.1136/annrheumdis-2019-eular.5860

\section{THU0479 SAFETY AND EFFICACY OF MEDICAL CANNABIS IN FIBROMYALGIA}

Iftach Sagy ${ }^{1}$, Lihi Bar-Lev Schleider ${ }^{2}$, Mahmoud Abu-Shakra ${ }^{1,2}$, Victor Novack ${ }^{2}$ ${ }^{1}$ Soroka Medical Center, Rheumatology, Beersheba, Israel; ${ }^{2}$ Soroka Medical Center, Beersheba, Israel

Background: Although chronic pain is a well established indication for medical cannabis therapy, there is scarce evidence to support the role of medical cannabis in the treatment of fibromyalgia.

Objectives: The aim of the study was to investigate the characteristics, safety and effectiveness of medical cannabis therapy for fibromyalgia. Methods: A prospective study with 6 months follow-up period based on fibromyalgia patients who were willing to answer questionnaire in a specialized medical cannabis clinic between 2015 and 2017.

Results: Among the 367 fibromyalgia patients the mean age was 52.9 \pm 15.1 , of whom $301(82.0 \%)$ were women. 28 patients (7.6\%) stopped the treatment prior to the six months follow-up. The six months response rate was $70.8 \%$. Pain intensity (scale $0-10$ ) reduced from a median of 9.0 at baseline to $5.0 \quad(\mathrm{p}<0.001)$, and 194 patients $(81.1 \%)$ achieved treatment response. In a multivariate analysis age above 60 years (Odds ratio $[\mathrm{OR}] 0.34,95 \%$ C.I $0.16-0.72$ ), concerns about cannabis treatment (OR $0.36,95 \%$ C.I $0.16-0.80$ ), spasticity (OR $2.26,95 \%$ C.I $1.08-4.72$ ) and previous use of cannabis (OR $2.4695 \%$ C.I 1.06-5.74) were associated with treatment outcome. The most common adverse effects were mild and included dizziness (7.9\%), dry mouth $(6.7 \%)$ and gastrointestinal symptoms $(5.4 \%)$.

Conclusion: Medical cannabis appears to be safe and effective alternative for the treatment of fibromyalgia symptoms. Standardization of treatment compounds and regimens are required.

Disclosure of Interests: : Iftach Sagy: None declared, Lihi Bar-Lev Schleider Employee of: employee of Tikun-Olam Ltd. without shares or options, Mahmoud Abu-Shakra: None declared, Victor Novack Consultant for: paid member of the Tikun Olam Ltd. scientific advisory board DOI: 10.1136/annrheumdis-2019-eular.111

\section{THU0480 IS PROLONGED SEDENTARY TIMEASSOCIATED WITH THE IMPACT OF THE DISEASE IN WOMEN WITH FIBROMYALGIA? THE AL-ÁNDALUS PROJECT}

Víctor Segura-Jiménez ${ }^{1}$, Blanca Gavilán Carrera ${ }^{2}$, Pedro Acosta-Manzano², Alberto Soriano Maldonado ${ }^{3,4}$, Inmaculada C Alvarez-Gallardo ${ }^{5}$,

Fernando Estevez-Lopez ${ }^{6,7}$. ${ }^{1}$ Faculty of Education Sciences, University of Cádiz, Department of Physical Education, Cádiz, Spain; ${ }^{2}$ Faculty of Sport Sciences, University of Granada, Department of Physical Education and Sports, Granada, Spain; ${ }^{3}$ Faculty of Education Sciences, University of Almería, Department of Education, Almería, Spain; ${ }^{4}$ CERNEP Research Center, University of Almería, Sport Research Group, CTS-1024, Almería, Spain; ${ }^{1}$ Faculty of Education Sciences, University of Cádiz, Department of Physical Education, Cádiz, Spain; ${ }^{6}$ Faculty of Sport Sciences, University of Granada, Deparment of Physical Education and Sports, Granada, Spain, ${ }^{7}$ University of Ulster, Belfast, United Kingdom

Background: Spending time seated has previously shown to be associated with worse symptomatology in fibromyalgia ${ }^{1}$. In addition, to accumulate sedentary time (ST) in prolonged, unbroken periods (bouts) has shown to be particularly harmful in the general population ${ }^{2}$.

Objectives: To examine the association of prolonged ST with the impact of the disease in women with fibromyalgia.

Methods: Four-hundred-and-seven $(51.4 \pm 7.6$ years old $)$ fibromyalgia women participated in this cross-sectional study. Sedentary time accumulated in bouts of $\geq 10, \geq 20, \geq 30$ and $\geq 60$ minutes, and moderate-to-vigorous physical activity (MVPA) were measured with triaxial accelerometry $\left(G T 3 X_{+}\right)$. To control for total ST, the percentage of ST spent in different bout categories were calculated (e.g., (time spent in bouts $>30 \mathrm{~min} /$ total ST) $\times 100$ ). We assessed different domains of fibromyalgia impact (function, overall, symptoms) and the global impact of fibromyalgia (total score) with the Revised Fibromyalgia Impact Questionnaire (FIQR). Separate linear regression models were built to assess the association of percentage of ST spent in different bout categories (independent variables) with the domains of fibromyalgia impact and the global impact of the disease (dependent variable) while controlling for age, fat percentage, medication for pain and depression, and total accelerometer wear time. Model 2 additionally included time spent in MVPA.

Results: Greater percentage of ST spent in bouts $\geq 10 \mathrm{~min}(\beta=0.171$, $p<0.001),>20 \min (\beta=0.163, p=0.001),>30 \min (\beta=0.169, p<0.001)$, and $\geq 60 \min (\beta=0.161, p=0.001)$ was associated with greater global impact of the disease. In model 2, greater percentage of ST spent in bouts $\geq 10$ $\min (\beta=0.145, p=0.003), \geq 20 \min (\beta=0.138, p<0.004), \geq 30 \min (\beta=0.146$, 